\title{
Upgrading and upstaging in prostate cancer: From prostate biopsy to radical prostatectomy
}

\author{
CAROLINA D'ELIA, MARIA ANGELA CERRUTO, ANTONIO CIOFFI, \\ GIOVANNI NOVELLA, STEFANO CAVALLERI and WALTER ARTIBANI \\ Department of Surgery, Urology Clinic, A.O.U.I. Verona, I-37134 Verona, Italy
}

Received January 27, 2014; Accepted June 17, 2014

DOI: $10.3892 / \mathrm{mco} .2014 .370$

\begin{abstract}
Prostate cancer $(\mathrm{CaP})$ is the most common malignancy in men and the second cause of cancer-related mortality after lung cancer. Several studies have evaluated the correlation between bioptic and pathological Gleason score (GS), documenting a correlation ranging between 30 and $60 \%$. The aim of this study was the evaluation of the association between bioptic and pathological GS in a series of patients undergoing prostate needle biopsy and subsequent radical prostatectomy. We also aimed to evaluate the possible prognostic factors of upgrading and upstaging. We prospectively collected and retrospectively reviewed data from 300 consecutive patients who underwent radical retropubic or robot-assisted prostatectomy at our Institution. Patients who underwent prostate needle biopsy, transrectal or transperineal, with a minimum of 5 samples, were included in this study. Upgrading and downgrading were defined as increase or decrease, respectively, from one prognostic grade group to another, similar to up- or downstaging. The mean age of the patients was 62.97 years and the mean prostate-spesific antigen (PSA) level was $7.83 \mathrm{ng} / \mathrm{ml}$. A total of $51.3 \%$ of the population underwent a transperineal prostate biopsy. The most frequently represented bioptic GS was $3+3(64.0 \%)$ followed by $3+4=7$ $(15.6 \%)$; the most frequent pathological Gleason score was $3+4(44.3 \%)$, followed by $3+3(31.0 \%)$. With reagard to the bioptic GS 4-5-6 group, approximately half of the specimens $(46.7 \%)$ were subsequently upgraded to GS $3+4$, and $5.3 \%$ to $4+3$. With regards to the bioptic GS $3+4$ group, $57.4 \%$ was confirmed in the surgical specimen. In the $4+3$ group, $23.5 \%$ of the cases was downgraded to $3+4$ and $35.3 \%$ was confirmed. With regards to stage, $\sim 39.7 \%$ of the patients received an upstaging on the pathological specimen. We evaluated the correlations between preoperative serum PSA level, prostate
\end{abstract}

Correspondence to: Dr Carolina D'Elia, Department of Surgery, Urology Clinic, A.O.U.I. Verona, 10 Piazzale L.A. Scuro, I-37134 Verona, Italy

E-mail: karolinedelia@gmail.com

Key words: prostate cancer, prostate biopsy, radical prostatectomy, upgrading, upstaging volume, digital rectal examination and biopsy type and none of the variables considered exhibited a correlation with any upgrading $(\mathrm{P}>0.05)$. Moreover, we evaluated the correlations between the aforementioned variables and upstaging and, at the multivariate analysis, only a serum PSA $<4 \mathrm{ng} / \mathrm{ml}$ was found to be an independent variable predictive of upstaging $(\mathrm{P}=0.017)$. Therefore, new tools are required to predict upgrading and upstaging of our patients, in order to ensure better counseling for optimal treatment planning.

\section{Introduction}

Prostate cancer $(\mathrm{CaP})$ is the most common malignancy in men and the second cause of cancer-related mortality after lung cancer (1). The diagnosis of $\mathrm{CaP}$ is mainly performed by digital rectal examination (DRE), serum prostate-spesific antigen (PSA) measurement and transperineal or transrectal ultrasound-guided biopsies. Prostate needle biopsy is one of the most common procedures performed in the common urological clinical practice; it is estimated that, in the U.S. alone, at least 800,000 prostate biopsies are performed annually (2).

Prostate biopsy has evolved over the years, starting from the technique described by Astraldi in 1937 (3) until the sextant prostate biopsy, described by Hodge et al in 1989 (4). Prostatic needle biopsy is currently considered the gold standard for the diagnosis of $\mathrm{CaP}$ and may be performed transrectally or transperineally, guided by ultrasound. The detection rates of these two techniques are comparable $(5,6)$ and there is no final consensus regarding the optimal number of samples, although the British Prostate Testing for Cancer and Treatment Study has recommended 10 core biopsies (7), with antibiotic therapy, commonly quinolone, under local anesthesia.

The International Society of Urological Pathology (ISUP) Conference was held in March, 2005 in San Antonio, Texas (8), during which a panel of international expert uropathologists updated the Gleason grading, in order to increase the reproducibility and reliability of the evaluation of the biopsy specimens. A correct assignment of the Gleason score (GS) may be crucial in terms of prognostic and therapeutic management of CaP. Several studies have assessed the effect of the ISUP Conference on the concordance of Gleason pattern and the possible change of prognostic group.

Billis et al (9) evaluated 172 patients who underwent prostate needle biopsy and subsequent radical prostatectomy and 
described a significant effect of the ISUP Conference modifications on the evaluation of the Gleason patterns and the resulting change in prognostic group. Following re-evaluation of the specimens, an increase by 1 or even 2 score points was observed in 16.8 and $0.6 \%$ of the cases, respectively.

Furthermore, $26.7 \%$ of the 'reassigned' patients had a higher preoperative PSA level, a larger tumor, more frequent positive surgical margins and higher-stage pathological disease. After the re-evaluation, a higher number of patients was assigned to the prognostic group of GS 8-10, exhibiting, at follow-up, a shorter time to biochemical disease recurrence (log-rank $\mathrm{P}=0.011)(9)$.

In a more recent study that evaluated 590 biopsy specimens according to the classic and modified GS, the number of cores with $75-100 \%$ pattern 4 cancer was increased by $95 \%$ (10). Over the last few years, the bioptic GS has become increasingly important, since several patients may be offered therapeutic alternatives to radical prostatectomy, such as active surveillance, and prostate biopsy results represent a crucial point in the management of the disease.

Several studies have evaluated the correlation between bioptic and pathological GS, documenting a correlation ranging between 30 and $60 \%$, particularly regarding low GS. The aim of this study was, therefore, to evaluate the association between bioptic and pathological GS in a series of patients undergoing prostate needle biopsy and subsequent radical prostatectomy and the possible prognostic factors of upgrading and upstaging.

\section{Materials and methods}

Patients. We prospectively collected and retrospectively reviewed data from 300 consecutive patients who underwent radical retropubic prostatectomy or laparoscopic robot-assisted prostatectomy at our Institution between January, 2010 and May, 2012. Patients who underwent prostate needle biopsy, transrectal or transperineal, at our Institution or at other centers, with a minimum of 5 samples, were included in this study.

Data collection. We collected clinical data regarding age, serum PSA level, free to total ratio, prostate volume calculated by transrectal ultrasound and clinical stage. Cases with neoadjuvant therapy were excluded from this analysis.

Surgical treatment. All the patients were subjected to radical prostatectomy within 6 months following prostate biopsy. The radical prostatectomy was performed by five operators. Retropubic prostatectomies were performed according to the technique described by Walsh and Donker (11) and robot-assisted laparoscopic prostatectomies were performed as previously described in the literature (12). Pelvic lymphadenectomy was performed in accordance with the indications of the Guidelines of the European Association of Urology (13).

Specimen grading. All the specimens were processed by two experienced uropathologists according to the TNM of 2009. The biopsies not performed at our center were not subjected to central review. Upgrading and downgrading were defined as an increase or decrease, respectively, from one prognostic grade group to another, similar to up- or downstaging. The grading prognostic groups were established according to the GS and grouped as follows: 5-6, 3+4, 4+3, 8 and 9-10.

Statistical analysis. Continuous variables were evaluated using mean and standard deviation or median and interquartile range, according to their distribution. The association between upgrading or upstaging and age, preoperative PSA level, PSA density, free to total ratio, number of positive samples and weight of the surgical specimen were evaluated using the Student's t-test or the Mann Whitney U test, depending on their distribution. The association between upgrading or upstaging and rectal findings, type and site of biopsy were evaluated using the Chi-square test, Student's t-test and multivariate logistic regression, as appropriate. Statistical analyses were performed using SPSS software, version 16.0 (SPSS Inc., Chicago, IL, USA).

\section{Results}

Clinicopathological characteristics. The clinical and pathological characteristics of the study population are summarized in Table I. The mean age of the patients was 62.97 years and $64.7 \%$ of patients were aged $<65$ years. The mean PSA level was $7.83 \mathrm{ng} / \mathrm{ml}$, with a free/total mean ratio of $12.66 \%$. Approximately $67 \%$ of the patients had a PSA level in the grey zone between 4 and $10 \mathrm{ng} / \mathrm{ml}$. The majority of the patients (79.7\%) presented with a negative DRE; $51.3 \%$ of the population underwent a transperineal prostate biopsy. Approximately half of the patients $(48.0 \%)$ underwent prostate biopsy at our Institution.

Upgrading and downgrading. The prostate volume was $<50 \mathrm{~cm}^{3}$ in $70.0 \%$ of the cases. The most frequently represented bioptic GS was $3+3(64.0 \%)$ followed by $3+4=7(15.6 \%)$, whereas the most frequent pathologic GS was $3+4(44.3 \%)$, followed by $3+3(31.0 \%)$. The surgical margins were negative in 227 patients. Table II shows the correspondence between bioptic and pathological GS. With regard to the bioptic GS 4-5-6 group, approximately half of the specimens (46.7\%) was subsequently upgraded to GS $3+4$, and $5.3 \%$ to $4+3$.

With regards to the bioptic GS $3+4$ group, $57.4 \%$ was confirmed in the surgical specimen. In the $4+3$ group, $23.5 \%$ of the cases were downgraded to $3+4$ and $35.3 \%$ were confirmed. With regards to the stage, $39.7 \%$ of the patients received an upstaging on the pathological specimen.

Correlation analysis. We evaluated the correlations between preoperative serum PSA level, prostate volume, DRE and biopsy type and none of the variables considered exhibited a correlation with any upgrading $(\mathrm{P}>0.05)$. We also evaluated the correlations between the aforementioned variables and upstaging and, at the multivariate analysis, only a serum PSA level $<4 \mathrm{ng} / \mathrm{ml}$ was found to be an independent variable predictive of upstaging (P 0.017) (Table III).

\section{Discussion}

The determination of the GS remains crucial in the evaluation of patients affected by $\mathrm{CaP}$ and in the management of this disease, 
Table I. Clinicopathological characteristics of the patients.

\begin{tabular}{|c|c|}
\hline Characteristics & Values \\
\hline \multicolumn{2}{|l|}{ Age, years } \\
\hline Mean & 62.97 \\
\hline Median & 63 \\
\hline Range & $41-77$ \\
\hline \multicolumn{2}{|l|}{ PSA, ng/ml } \\
\hline Mean & 7.83 \\
\hline Median & 6.1 \\
\hline Range & $0.6-57.4$ \\
\hline \multicolumn{2}{|l|}{ Free/total ratio, $\%$} \\
\hline Mean & 12.66 \\
\hline Median & 11 \\
\hline Range & $2-48$ \\
\hline \multicolumn{2}{|l|}{ Volume, $\mathrm{cm}^{3}$} \\
\hline Mean & 42.69 \\
\hline Median & 40 \\
\hline Range & $13-120$ \\
\hline \multicolumn{2}{|c|}{ PSA density, $\mathrm{ng} / \mathrm{ml} / \mathrm{cm}^{3}$} \\
\hline Mean & 0.20 \\
\hline Median & 0.15 \\
\hline Range & $0.02-1.4$ \\
\hline \multicolumn{2}{|c|}{ Clinical stage, no. (\%) } \\
\hline cT1c & $239(79.7)$ \\
\hline $\mathrm{T} 2 \mathrm{a}-\mathrm{b}$ & $36(12.0)$ \\
\hline cT2c & $25(8.3)$ \\
\hline \multicolumn{2}{|l|}{ Biopsy, no. (\%) } \\
\hline Transperineal & $154(51.3)$ \\
\hline Transrectal & $146(48.7)$ \\
\hline \multicolumn{2}{|l|}{ Biopsy site, no. (\%) } \\
\hline Our institution & $144(48.0)$ \\
\hline Other institution & $156(52.0)$ \\
\hline \multicolumn{2}{|l|}{ Cores, no. } \\
\hline Mean & 13.57 \\
\hline Median & 14 \\
\hline Range & $5-28$ \\
\hline \multicolumn{2}{|l|}{ Positive cores, no. } \\
\hline Mean & 4.15 \\
\hline Median & 3 \\
\hline Range & $1-15$ \\
\hline \multicolumn{2}{|c|}{ Bioptic Gleason score, no. (\%) } \\
\hline NA & $5(1.7)$ \\
\hline 4 & $2(0.7)$ \\
\hline 5 & $14(4.7)$ \\
\hline $3+3$ & $192(64.0)$ \\
\hline $3+4$ & $47(15.6)$ \\
\hline $4+3$ & $17(5.7)$ \\
\hline 8 & $20(6.6)$ \\
\hline $9-10$ & $3(1.0)$ \\
\hline \multicolumn{2}{|l|}{ Specimen weight, g } \\
\hline Mean & 56.91 \\
\hline Median & 52 \\
\hline Range & $20-140$ \\
\hline
\end{tabular}

Table I. Continued.

\begin{tabular}{lc}
\hline Characteristics & Values \\
\hline Pathological Gleason score, no. $(\%)$ & \\
NA & $5(1.7)$ \\
5 & $3(1.0)$ \\
$3+3$ & $93(31.0)$ \\
$3+4$ & $133(44.3)$ \\
$4+3$ & $23(7.6)$ \\
8 & $26(8.7)$ \\
$9-10$ & $17(5.7)$ \\
Surgical margins, no. $(\%)$ & \\
Negative & $227(76.0)$ \\
Positive & $73(24.0)$
\end{tabular}

PSA, prostate-specific antigen; NA, not available.

which may range from an active surveillance protocol to radical prostatectomy and multimodal therapies. Several previous studies have analyzed the correlation between the biopsy evaluation and the results obtained from surgical specimens.

The comparisons between the results of the histological biopsy compared to the surgical specimen exhibited a correlation equal to $\sim 50 \%$ (14-17). The results of the prostate biopsy and the surgical specimen may differ for several reasons, such as incorrect evaluation by the pathologist, sampling errors and the presence of borderline grading. Several studies demonstated that a higher number of cores, compared to the sextant biopsy, may lead to a lower percentage of upgrading.

Capitanio et al (18) evaluated a series of 301 patients with low-risk CaP according to the D'Amico criteria (19) (clinical stage T1c - T2a, PSA $<10 \mathrm{ng} / \mathrm{ml}$ and biopsy GS 6) that underwent extended prostate biopsy (median number of cores, 18) and subsequent radical prostatectomy. The GS agreement between biopsies and surgical specimens was 47.5\% (143 patients), while upgrading was recorded in 38.5\% cases (116 patients), 31.9\% of which (96 patients) presented with a significant upgrade to $\mathrm{GS} \geq 7$. In patients evaluated with 10-12 core biopsies, the upgrading was $47.9 \%$, compared to 31.6 and $23.5 \%$ with $13-18$ or $>18$ cores, respectively, with a statistically significant P-value, demonstrating that a larger sampling of the gland may avoid subsequent upgrading and may help in planning an appropriate treatment approach.

In our series, however, variables predictive of upgrading were not detected and the type of biopsy or the number of samples taken were not identified as predictors of upgrading. In 2012, Epstein et al (20) evaluated the largest series in the literature, with 7,643 patients, analysing the correlation between bioptic and definitive GS. Of the patients with GS 5-6, 36.3\% underwent an upgrading and $20 \%$ of the patients exhibited a tertiary Gleason pattern. In half of the remaining cases, there was an equal proportion of up- and downgrading. Half of the cases had matching GS 3+4=7 at biopsy and RP, with an approximately equal number of cases down- and upgraded at RP.

A bioptic GS 8 led to an almost equal distribution between RP GS $4+3=7,8$ and $9-10$. A total of $58 \%$ of the cases had 
Table II. Upgrading and downgrading.

\begin{tabular}{|c|c|c|c|c|c|c|c|c|c|c|c|c|c|c|}
\hline \multirow{2}{*}{$\begin{array}{l}\text { Bioptic } \\
\text { GS }\end{array}$} & \multicolumn{2}{|c|}{$4-5-6$} & \multicolumn{2}{|c|}{$3+4=7$} & \multicolumn{2}{|c|}{$4+3=7$} & \multicolumn{2}{|c|}{8} & \multicolumn{2}{|c|}{$9-10$} & \multicolumn{2}{|c|}{ NA } & \multicolumn{2}{|c|}{ Total } \\
\hline & no. & $\%$ & no. & $\%$ & no. & $\%$ & no. & $\%$ & no. & $\%$ & no. & $\%$ & no. & $\%$ \\
\hline \multicolumn{15}{|l|}{ RP GS } \\
\hline 0 -NA & 3 & 1.4 & 1 & 2.1 & 0 & 0.0 & 0 & & 0 & & 1 & 20.0 & 5 & 1.7 \\
\hline $5-6$ & 92 & 44.2 & 2 & 4.3 & 0 & 0.0 & 0 & & 0 & & 2 & 40.0 & 96 & 32.0 \\
\hline $3+4=7$ & 97 & 46.7 & 27 & 57.4 & 4 & 23.5 & 3 & 15.0 & 0 & & 2 & 40.0 & 133 & 44.3 \\
\hline $4+3=7$ & 11 & 5.3 & 5 & 10.7 & 6 & 35.3 & 1 & 5.0 & 0 & & 0 & 0.0 & 23 & 7.6 \\
\hline 8 & 3 & 1.4 & 9 & 19.1 & 5 & 29.4 & 8 & 40.0 & 1 & 33.3 & 0 & 0.0 & 26 & 8.7 \\
\hline $9-10$ & 2 & 1.0 & 3 & 6.4 & 2 & 11.8 & 8 & 40.0 & 2 & 66.7 & 0 & 0.0 & 17 & 5.7 \\
\hline Total & 208 & 100.0 & 47 & 100.0 & 17 & 100.0 & 20 & 100.0 & 3 & 100.0 & 5 & 100.0 & 300 & 100.0 \\
\hline
\end{tabular}

GS, Gleason score; RP, radical prostatectomy; NA, not available.

Table III. Upstaging multivariable analysis.

\begin{tabular}{lccc}
\hline Variables & SE & P-value & $95 \%$ CI \\
\hline $\begin{array}{l}\text { Age, years } \\
\leq 65\end{array}$ & 0.259 & 0.280 & $0.455-1.256$ \\
$\begin{array}{l}\text { DRE } \\
\text { PSA, ng/ml }\end{array}$ & 0.302 & 0.655 & $0.633-2.071$ \\
$<4$ & & & \\
$>4$ & 0.372 & $0.017^{\mathrm{a}}$ & $1.171-5.027$ \\
Volume $<50$ cc & 0.333 & 0.877 & $0.495-1.824$ \\
Biopsy & 0.270 & 0.625 & $0.673-1.936$ \\
TP & & & \\
TR & 0.378 & 0.997 & $0.477-2.100$ \\
Bio $=$ trans & 0.273 & 0.795 & $0.545-1.591$ \\
\end{tabular}

a Statistically significant. SE, standard error; CI, confidence interval; DRE, digital rectal exploration; PSA, prostate-specific antigen; TP, transperineal; TR, transrectal.

matching GS 9-10 at biopsy and RP. In addition, at the multivariate analysis, the authors reported that increasing age $(\mathrm{P}<0.0001)$, increasing serum prostate-specific antigen level $(\mathrm{P}<0.0001)$, decreasing RP weight $(\mathrm{P}<0.0001)$ and increasing maximum percentage cancer/core $(\mathrm{P}<0.0001)$ predicted the upgrading from a bioptic GS 5-6 to a higher one at RP (20). In our series, the presence of tertiary pattern was not assessed; therefore, we could not perform an evaluation regarding the presence and possibility of up- or downgrading.

In our study, with regards to GS $4-5-6,44.2 \%$ of cases matched, while there was an upgrading in $54.4 \%$ of the cases. With pattern $3+4$, there was a correlation between bioptic and definitive GS in $57.4 \%$ of the cases, downgrading in $6.4 \%$ of the cases and upgrading in $36.2 \%$, a higher percentage when compared to the data presented by our colleagues. With GS $4+3$, concordance was obtained in $35.3 \%$ of cases and a preponderance of upgrading $(41.2 \%)$ compared to the downgrading $(23.5 \%)$, an even higher percentage if compared to the data presented by Epstein et al (20). GS 8 was matched in $40 \%$ and GS 9-10 in $66.7 \%$ of the cases, almost overlapping with the data presented by our colleagues.

Epstein et al (20) also conducted a wide literature review, selecting only studies that evaluated series of patients with numerosity $>100$, documenting the presence of GS upgrading from 6 to 7 in 3,975 out of 11,472 cases $(35 \%)$, with a mean percentage of $36.0 \%$ and a median of $35.5 \%$, with results ranging from 14 to $51 \%$ (21-23). In our series, none of the analyzed variables was identified as an independent predictor of up- or downgrading, whereas a PSA level $<4 \mathrm{ng} / \mathrm{ml}$ was found to be predictive of upstaging. In the literature, as in our study, age was not found to be a variable correlated with upgrading or upstaging, whereas age was found to be predictive in the study of Epstein et al (20), with a difference between the 2 groups of only 1.6 years.

PSA values, in contrast to our data, are often found to be predictive of upgrading, albeight with weak associations. According to our data, a preoperative PSA $<4 \mathrm{ng} / \mathrm{ml}$ was found to be an independent predictor of upstaging; this highlights the questions regarding cancer as an incidental finding, which, even with low PSA levels, is found to be significant, as already suggested by Thompson et al (24). Moreover, in the literature, an increase in prostate volume was found to be less predictive of upgrading, while in the study of Epstein et al a decrease was documented in the upgrading ratio in prostate glands weighing $>75 \mathrm{~g}$.

Approximately half of the studies available in the literature documented an association between the percentage of cores invaded by cancer and upgrading; however, in this study, we did not consider this variable. The significance of the ability to predict a possible upgrading or upstaging of prostate biopsy lies with the risk of 'falsely' estimating a low GS, which may lead to the selection of a treatment not adequately aggressive, potentially putting the patient at risk of poor oncologic outcomes. For this reason, Serkin et al (25) retrospectively evaluated 2,884 patients who underwent radical prostatectomy, documenting an upgrading in $36.8 \%$ of patients with a bioptic GS 6 . The authors of that study also demonstrated that the status of the surgical margins, capsule, seminal vesicles and lymph node involvement were more favorable in patients 
with a GS correlation between bioptic and surgical specimens $(\mathrm{P}<0.0001)$.

Moreover, patients with lower prostate volume had a higher PSA density and an increased risk of upgrading. The Kaplan-Meier curves suggested that patients with upgrading were at an increased risk of biochemical disease recurrence compared to patients with a correlation between the specimens $(\mathrm{P}<0.0001)$ and a higher risk to undergo salvage hormone therapy $(\mathrm{P}<0.0001)$.

Our study had several limitations, as follows: the design of the study was retrospective and over half of the biopsies were not performed in our Institution; we did not perform a centralized review of the specimens, with a variable number of biopsies and with the inability to evaluate, in some cases, the percentage of cores involved; we do not have an oncological follow-up of the patients and, therefore, we cannot correlate the biopsy results with the follow-up of the patients.

The upgrading and upstaging from prostate biopsy to radical prostatectomy is an important topic of discussion and may be of significant value at the clinical level, for treatment planning, as well as for the prediction of cancer outocomes. Therefore, new tools are required to predict upgrading and upstaging of our patients, in order to ensure better counseling for optimal treatment planning.

\section{References}

1. Hanno P, Malkowicz SB and Wein AJ (eds): Clinical Manual of Urology. 3rd edition. McGraw-Hill, New York, NY, pp519, 2001.

2. Maccagnano C, Scattoni V, Roscigno M, et al: Anaesthesia in transrectal prostate biopsy: which is the most effective technique? Urol Int 87: 1-13, 2011.

3. Astraldi A: Diagnosis of cancer of the prostate: biopsy by rectal route. Urol Cutaneous Rev 41: 421-422, 1937.

4. Hodge KK, McNeal JE, Terris MK and Stamey TA: Random systematic versus directed ultrasound guided transrectal core biopsies of the prostate. J Urol 142: 71-74, discussion 74-75, 1989

5. Hara R, Jo Y, Fujii T, et al: Optimal approach for prostate cancer detection as initial biopsy: prospective randomized study comparing transperineal versus transrectal systematic 12-core biopsy. Urology 71: 191-195, 2008.

6. Takenaka A, Hara R, Ishimura T, et al: A prospective randomized comparison of diagnostic efficiency between transperineal and transrectal 12-core prostate biopsy. Prostate Cancer Prostatic Dis 11: 134-138, 2008.

7. Donovan J, Hamdy F, Neal D, et al; ProtecT Study Group: Prostate testing for cancer and treatment (ProtecT) feasibility study. Health Technol Assess 7: 1-88, 2003.

8. Epstein JI, Allsbrook WC Jr, et al; ISUP Grading Committee: The 2005 International Society of Urological Pathology (ISUP) Consensus Conference on Gleason Grading of Prostatic Carcinoma. Am J Surg Pathol 29: 1228-1242, 2005.

9. Billis A, Guimaraes MS, Freitas LL et al: The impact of the 2005 International Society of Urological Pathology consensus conference on standard Gleason grading of prostatic carcinoma in needle biopsies. J Urol 180: 548-553, 2008.
10. Delahunt B, Lamb DS, Srigley JR, et al: Gleason scoring: a comparison of classical and modified (International Society of Urological Pathology) criteria using nadir PSA as a clinical end point. Pathology 42: 339-343, 2008.

11. Walsh PC and Donker PJ: Impotence following radical prostatectomy: insight into etiology and prevention. J Urol 128: 492-497, 1982.

12. Ficarra V, Novara G, Fracalanza S, et al: A prospective, non-randomized trial comparing robot-assisted laparoscopic and retropubic radical prostatectomy in one European institution. BJU Int 104: 534-539, 2009.

13. Heidenreich A, Bellmunt J, Bolla M, et al: EAU guidelines on prostate cancer. Part 1: screening, diagnosis, and treatment of clinically localised disease. Eur Urol 59: 61-71, 2011.

14. Mian BM, Lehr DJ, Moore CK, et al: Role of prostate biopsy schemes in accurate prediction of Gleason scores. Urology 67: 379-383, 2006.

15. Sengupta S, Slezak JM, Blute ML, et al: Trends in distribution and prognostic significance of Gleason grades on radical retropubic prostatectomy specimens between 1989 and 2001. Cancer 106: 2630-2635, 2006.

16. Melia J, Moseley R, Ball RY, et al: A UK-based investigation of inter- and intra-observer reproducibility of Gleason grading of prostatic biopsies. Histopathology 48: 644-654, 2006.

17. Kvåle R, Møller B, Wahlqvist R, et al: Concordance between Gleason scores of needle biopsies and radical prostatectomy specimens: a population-based study. BJU Int 103: 1647-1654, 2009.

18. Capitanio U, Karakiewicz PI, Valiquette L, et al: Biopsy core number represents one of foremost predictors of clinically significant Gleason sum upgrading in patients with low-risk prostate cancer. Urology 73: 1087-1091, 2009.

19. D'Amico AV, Whittington R, Malkowicz SB, et al: Biochemical outcome after radical prostatectomy, external beam radiation therapy, or interstitial radiation therapy for clinically localized prostate cancer. JAMA 280: 969-974, 1998

20. Epstein JI, Feng Z, Trock BJ and Pierorazio PM: Upgrading and downgrading of prostate cancer from biopsy to radical prostatectomy: incidence and predictive factors using the modified Gleason grading system and factoring in tertiary grades. Eur Urol 61: 1019-1024, 2012

21. Budaus L, Graefen M, Salomon G, et al: The novel nomogram of Gleason sum upgrade: possible application for the eligible criteria of low dose rate brachytherapy. Int J Urol 17: 862-868, 2010.

22. Colleselli D, Pelzer AE, Steiner E, et al: Upgrading of Gleason score 6 prostate cancers on biopsy after prostatectomy in the low and intermediate tPSA range. Prostate Cancer Prostatic Dis 13: 182-185, 2010.

23. Ruijter E, van Leenders G, Miller G, et al: Errors in histological grading by prostatic needle biopsy specimens: frequency and predisposing factors. J Pathol 192: 229-233, 2000.

24. Thompson IM, Pauler DK, Goodman PJ, et al: Prevalence of prostate cancer among men with a prostate-specific antigen level $<$ or = 4 ng per milliliter. N Engl J Med 350: 2239-2246, 2004.

25. Serkin FB, Soderdahl DW, Cullen J, et al: Patient risk stratification using Gleason score concordance and upgrading among men with prostate biopsy Gleason score 6 or 7 . Urol Oncol 28: 302-307, 2010. 